\title{
RANDOM PERTURBATIONS OF SINGULAR SPECTRA
}

\author{
JAMES S. HOWLAND
}

(Communicated by Palle E. T. Jorgensen)

\begin{abstract}
The singular parts of the self-adjoint operators $T$ and $H=T+V$ are mutually singular for "almost every" bounded perturbation $V$.
\end{abstract}

Let $T$ and $H=T+V$ be self-adjoint operators on a separable Hilbert space $\mathscr{H}$. In 1965, Donaghue [1] proved the following theorem.

Theorem 1. If $V=c\langle\cdot, \varphi\rangle \varphi$ is of rank one, and $\varphi$ is $T$-cyclic, then the singular parts of $T$ and $H$ are supported on disjoint sets.

We shall generalize this result by proving that the singular parts of $T$ and $H=T+V$ are mutually singular for "almost every" bounded perturbation $V$. This theorem, which follows easily by the methods of Simon-Wolff [5] and the author [2], illustrates an essential instability of the singular spectrum.

Let $T$ be self-adjoint, $\varphi_{n}$ an arbitrary complete orthonormal set, $c_{n}>0$ an arbitrary bounded sequence of positive numbers, and $X_{n}(\omega)$ a sequence of independent random variables, uniformly distributed on $[-1,1]$. Define

$$
H(\omega)=T+V(\omega),
$$

where

$$
V(\omega)=\sum_{n=1}^{\infty} c_{n} X_{n}(\omega)\left\langle\cdot, \varphi_{n}\right\rangle \varphi_{n} .
$$

Theorem 2. The singular parts $T$ and $H(\omega)$ are supported on disjoint sets, almost surely.

Proof. We sketch the proof, which follows [2]. Let $N$ be a set of Lebesgue measure zero which supports the singular part of $T$. Define the multiplication operator

$$
\mathbf{H} u(\omega)=H(\omega) u(\omega)
$$

on $L_{2}(\Omega, P ; \mathscr{H})$.

Lemma. $\mathbf{H}$ is spectrally absolutely continuous.

Received by the editors February 5, 1990.

1980 Mathematics Subject Classification (1985 Revision). Primary 47A55, 60H25.

Supported by NSF Contract DMS-8801548. 
Assuming this for the moment, and taking $u(\omega)=u$ to be a constant function, we have

$$
0=|\mathbf{E}[N] u|^{2}=\int_{\Omega}\left|E_{\omega}[N] u\right|^{2} P(d \omega),
$$

and hence

$$
E_{\omega}[N] u=0, \text { a.s. }
$$

Letting $u$ range over a countable dense set gives

$$
E_{\omega}[N]=0 \text {, a.s., }
$$

which is the result. (This argument is essentially due to Kotani.)

The following proof of the lemma is slightly more elementary than that of [2], so we have included it here.

Proof. Let $Q$ be the coordinate operator of multiplication by $x$ on $L^{2}(\mathbf{R})$ and

$$
P=-i \frac{d}{d x}
$$

its conjugate. If $f(t)$ is a bounded smooth function with $f^{\prime}(t)>0$ (for example, $\arctan (t))$, then because, under the Fourier transformation

$$
Q=i \frac{d}{d p}
$$

we have

$$
i[f(P), Q]=f^{\prime}(P) \geq 0 .
$$

If $\chi(x)$ is the characteristic function of $[-1,1]$, then

$$
i[\chi(Q) f(P) \chi(Q), \chi(Q) Q]=\chi(Q) f^{\prime}(P) \chi(Q)>0 .
$$

Thus, the operator $X=\chi(Q) Q$ of multiplication by $x$ on $L^{2}[-1,1]$ has a positive commutator with the bounded operator,

$$
B=\chi(Q) f(P) \chi(Q) \text {. }
$$

Now, representing $\Omega$ as the infinite product of $[-1,1]$ 's, we have that

$$
L^{2}(\Omega ; \mathscr{H})=\left(\bigotimes_{n=1}^{\infty} L^{2}[-1,1]\right) \otimes \mathscr{H} .
$$

Each $X_{n}(\omega)$ becomes multiplication by $\chi(Q) Q$ on one factor of the tensor product. Let $B_{n}$ be as above with

$$
i\left[B_{n}, X_{n}\right]>0 \text {, }
$$

and define

$$
\mathbf{A}=\sum_{n=1}^{\infty} \frac{1}{2^{n}} B_{n}
$$

Then

$$
i[\mathbf{A}, \mathbf{H}]=i \sum_{n=1}^{\infty} c_{n}\left[B_{n}, X_{n}\right] \otimes\left\langle\cdot, \varphi_{n}\right\rangle \varphi_{n} \geq 0
$$


Hence, $\mathbf{H}$ has a positive commutator with a bounded operator of dense range and is therefore absolutely continuous by the Kato-Putnam theorem [4, p. 157].

Remarks. (1) One could replace the uniform distribution by other bounded, absolutely continuous distributions [2, p. 67]. In fact, as long as $X_{n}$ are independent, one could presumably relax the requirement that they be identically distributed.

(2) One can take $c_{j}$ tending very rapidly to zero, or not tending to zero at all, so that $V(\omega)$ is a very general sort of diagonal operator. This is rather satisfactory if one recalls that, by the Weyl-von Neumann Theorem [3, p. 523], every bounded self-adjoint operator differs from such an operator by an operator of arbitrarily small Hilbert-Schmidt norm.

\section{REFERENCES}

1. W. F. Donoghue, On the perturbation of spectra, Comm. Pure Appl. Math. 18 (1965), 559579.

2. J. S. Howland, Perturbation theory of dense point spectra, J. Funct. Anal. 74 (1987), 52-80.

3. T. Kato, Perturbation Theory for linear operators, Springer-Verlag, New York, 1966.

4. M. Reed and B. Simon, Methods of modern mathematical physics. II, Academic Press, New York, 1978.

5. B. Simon and T. Wolff, Singular continuous spectrum under rank one perturbations and localization for random Hamiltonians, Comm. Pure Appl. Math. 39 (1986), 75-90.

Department of Mathematics, University of Virginia, Charlottesville, Virginia 22903 